\title{
LIGHT SCATTERING BY PARTICLES WITH ARBITRARY SHAPE IN THE VICINITY OF THE BACKWARD SCATTERING DIRECTION WITHIN GEOMETRICAL OPTICS APPROXIMATION
}

\author{
Victor Shishko*, Alexander Konoshonkin, Natalia Kustova, Anatoli Borovoi, Dmitry Timofeev
}

\author{
V.E. Zuev Institute of Atmospheric Optics SB RAS, Tomsk, 634055, Russia
}

*Email:sva@iao.ru

\begin{abstract}
The work presents the solution for the light scattering problem by arbitrarily-shaped particles in the vicinity of the backward scattering direction. The solution was obtained within the framework of the geometrical optics approximation. The refractive index was equal to 1.3116 . It was shown that the general contribution of scattering light for arbitrarily-shaped particles in the vicinity of the backscattering direction consists of the specular reflection of the particles and two types of non-specular optical beams. It is shown that the optical characteristics of the ice particles with arbitrary shapes correspond to experimental data.
\end{abstract}

\section{INTRODUCTION}

Despite the fact that attempts to solve the inverse problem of laser polarization sensing of ice clouds have been made for a long time [1], this problem has not been solved yet. The main difficulty in interpreting experimentally obtained lidar data is the absence of an effective method for solving the direct problem of light scattering on ice crystal particles typical for real cirrus clouds.

\section{METHODOLOGY}

To build such a solution, the physical optics approximation was developed in the V.E. Zuev Institute of Atmospheric Optics SB RAS [2]. This method is effective for solving the direct problem of light scattering by crystal particles of cirrus clouds ranging in size from 10 to $1000 \mu \mathrm{m}$ [3]. Using this method, the Mueller matrices database for the main types of atmospheric ice crystals (hexagonal plates and columns, droxtals, bullets, deformed particles, and aggregates) was calculated. The work on the theoretical calculation of Mueller matrices for all this variety of forms and sizes of atmospheric ice crystals has been going on for many years. The database is available at the IOA SB RAS ftp server [4]. However, the most traditional shape of ice crystals reveal some spectral dependence in their optical characteristics, which is not observed in experiments.

To overcome this discrepancy, some more complicated shapes of the ice crystals should be considered. In this contribution, the differential cross sections for arbitrarily shaped particles have been calculated within the geometrical optics approximation.

\section{RESULTS}

The full Mueller matrices have been calculated for a set of 50 particle shapes with random orientations. The Maximal dimensions of all particles were approximately equal to $20 \mu \mathrm{m}$. As an example, Fig. 1 presents the results of the calculation of the differential scattering cross section (DSCS) of these shapes. The refractive index was taken equal to 1.3116 .

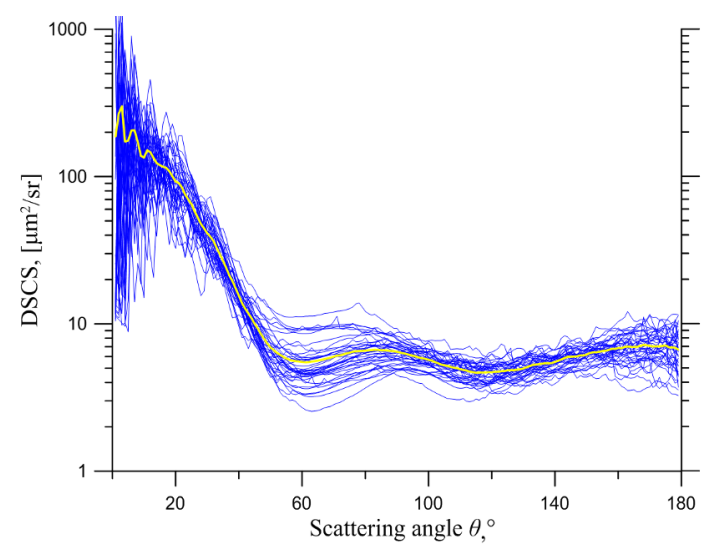

Figure 1. Differential scattering cross section obtained for a set of 50 ice particles of arbitrary shape. The yellow line - the average value over the entire set of particles

For a preliminary analysis, five particles those DSCS scarcely deviated from the average values in the vicinity of backward scattering direction $\left(\theta=180^{\circ}\right)$ in Fig. 1 were chosen. These particles and their optical properties are shown in Fig. 2 and table 1. It can be seen that the optical characteristics of these five ice particles are in the 
range of experimentally observed values $[5,6]$, despite the fact that such particles are not typical for cirrus clouds.

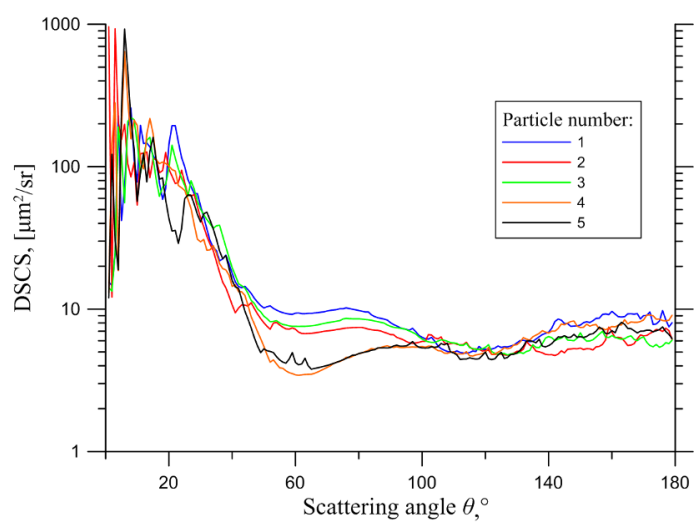

Figure 2. Differential scattering cross section for chosen five ice particles of arbitrary shape

Despite the fact that the selected particles differ in the values of their optical characteristics over almost the entire range of scattering angles, in the vicinity of the backward scattering direction, these particles have similar values of the optical characteristics. Therefore, it is sufficient to consider in more detail only one particle, for example, the particle of number 5 .

Table 1. Five particles of arbitrary shape, their appearance and optical characteristics in the backscattering direction

\begin{tabular}{|c|c|c|c|}
\hline $\begin{array}{c}\text { Particle } \\
\text { number }\end{array}$ & Appearance & LR, sr & DR \\
\hline 1 & & 51.26 & 0.51 \\
\hline 2 & & 55.72 & 0.52 \\
\hline 3 & & 62.1 & 0.47 \\
\hline 4 & & 38.12 & 0.54 \\
\hline 5 & 51.57 & 0.51 \\
\hline \multicolumn{2}{|c|}{ Average value } & 50.58 & 0.51 \\
\hline
\end{tabular}

For the particle of number 5, the DSCS in the vicinity of the backward direction is formed due to specular reflection from the particle and about 2200 non-specular beams that occurred in the backward scattering direction, Fig. 3. Each nonspecular beam is characterized by its own trajectory.

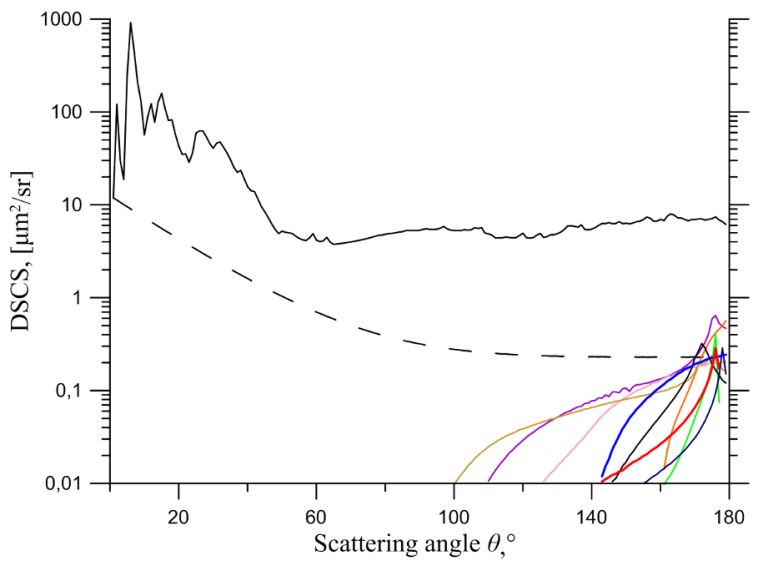

Figure 3. Differential scattering cross section for the particle of number 5 and the contributions of some typical non-specular beams (dotted line is the contribution of specular reflection, color lines were the contribution of various non-specular beams)

To differentiate the non-specular beams, distributions of light intensities over the backward scattering semi-sphere have been calculated for any beams (Figs. 4 and 6). The intensities are drawn as the dots of different brightness at a fixed particle orientation. A set of the dots shown in Figs. 4 and 6 gives 2D distributions of scattered intensities over the backward semi-sphere for a randomly oriented particle where rotation around the incident direction is excluded.

As a result, all non-specular trajectories occurred in the backward scattering directions can be divided into two types: the trajectories having a uniform distribution in the vicinity of the backward scattering direction (Fig. 4) and trajectories with uneven distribution (Fig. 6), concentrated in an area close to the backscattering direction.

Consider the non-specular beams with a uniform distribution in the vicinity of the backward scattering direction on the example of the nonspecular beam highlighted by blue in Fig. 3. The 
ray trajectories for this non-specular beam are shown in Figure 5.

The non-specular beam that corresponds to Figs. 4 and 5 gives a high depolarization ratio close to 0.5 in backscattering. Exactly these beams form the main contribution in backscattering when calculating within geometrical optics approximation.

Further, it is worth to consider the non-specular beams with uneven distribution concentrated in an area close to the backscattering direction, for example, the non-specular beam highlighted by red in Fig. 3. The trajectory of rays for this nonspecular beam is shown in Fig. 7.

a)

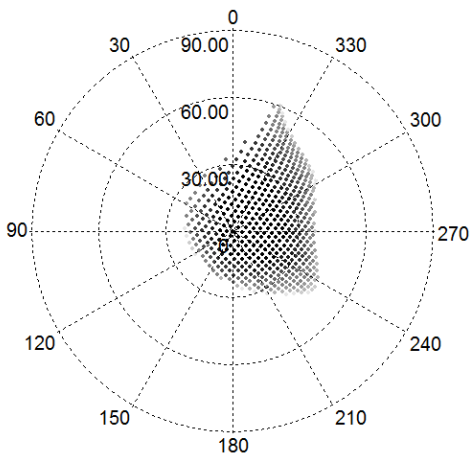

b)

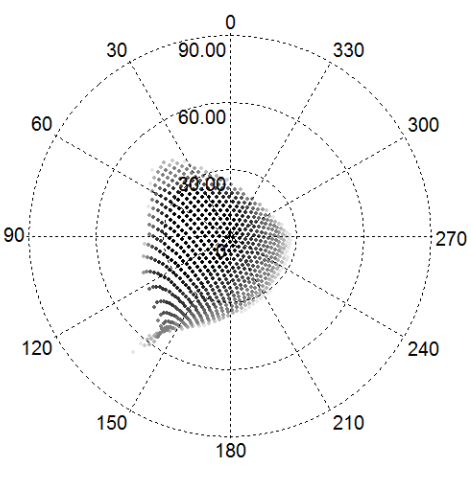

Figure 4. Backscattering semi-sphere, the point in the center of semi-sphere corresponds to the backscattering point: a) - the distribution of the non-specular beam in backscattering direction

(highlighted by blue in Fig. 3); b) - the distribution of the reciprocal non-specular beam

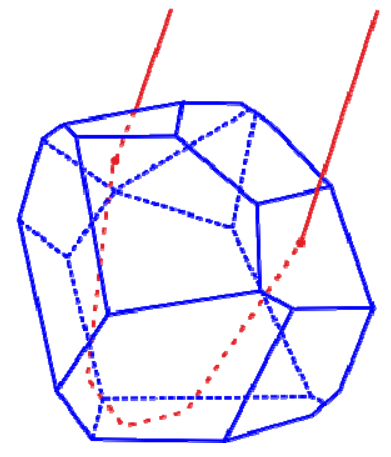

Figure 5. Visualization of non-specular trajectory presented in Fig. 4

However the non-specular beam presented in Fig. 6 and Fig. 7 is noteworthy because of the fact that it gives an insignificant contribution in backscattering in any calculations within geometrical optics approximation. Consequently, it is necessary to take these trajectories into account within the physical optics approximation because these beams would give a noticeable contribution by diffraction rings in backscattering.

a)

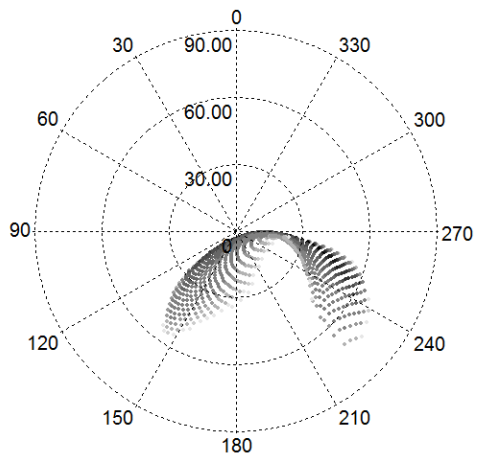

b)

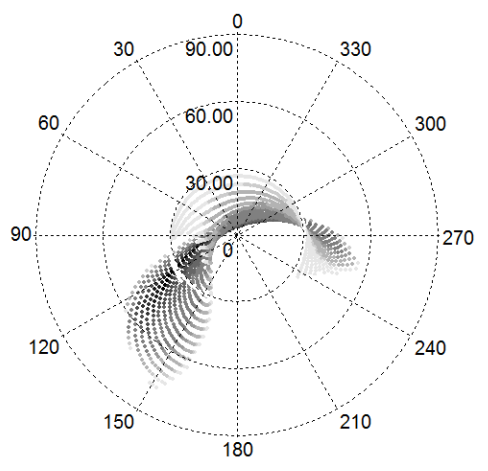

Figure 6. a) - The same as Fig. 4, but for the optical beam highlighted by red in Fig. 3; b) - the distribution of the reciprocal non-specular beam 


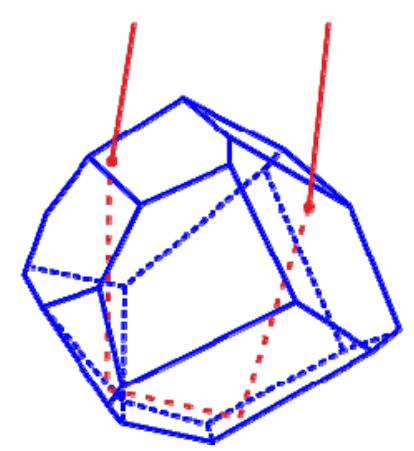

Figure 7. Visualization of non-specular trajectory presented in Fig. 6

\section{CONCLUSION}

The arbitrarily shaped particles without particularity in backward scattering direction were analyzed. It was shown that the main contribution in the vicinity of backscattering direction for such kind of particles is formed by both specular reflection and two types of beams described in Section 3. Also it was shown that their optical characteristics approximately correspond to the experimental data.

For further calculating the optical characteristics of this kind of particles it is necessary to take into account the fact that a part of optical beams would give a contribution by diffraction in backscattering.

\section{ACKNOWLEDGEMENTS}

This work is supported by the Russian Foundation for Basic Research under Grants no. 18-05-00568, no. 18-55-53046, and no. 19-01-00351, in part by the President of the Russian Federation MD3306.2019.5. The calculation of the irregular particle is supported only by Russian Science Foundation (18-77-10035).

\section{REFERENCES}

[1] D. N. Romashov, B. V. Kaul, I. V. Samokhvalov, Atmospheric and oceanic optics. 13(09), 794-800 (2000)

[2] A. V. Konoshonkin, N. V. Kustova, A. G. Borovoi, D. N. Timofeev, V. A. Shishko, Proc. SPIE. 10466, 104662C (2017)

[3] Z. Wang, V. A. Shishko, A. V. Konoshonkin, N. V. Kustova, A. G. Borovoi, G. G. Matvienko, C. Xie, D.
Liu, Y. Wang, Atmospheric and Oceanic Optics. 30(3), 234-235 (2017)

[4] URL: ftp://ftp.iao.ru/pub/GWDT/Physical_optics/B ackscattering/

[5] W.-N. Chen, C.-W. Chiang, J.-B. Nee, Appl. Opt. 41, 6470-6476 (2002)

[6] R. Kuehn, R. Holz, E. Eloranta, M. Vaughan, J. Hair, EPJ Web of Conferences. 119 (2016) doi:10.1051/epjconf/201611916009 